\title{
Conventional chemoradiation vs. induction chemotherapy followed by conventional chemoradiation for locally advanced head and neck cancer: A prospective, randomized study
}

\author{
AMIT RANA $^{1}$, PRIYANKA RANA ${ }^{2}$, MANOJ GUPTA $^{3}$, RAJEEV SEAM $^{4}$ and MANISH GUPTA ${ }^{1}$ \\ ${ }^{1}$ Department of Radiotherapy, Regional Cancer Centre, Indira Gandhi Medical College, Shimla, \\ Himachal Pradesh 171 001; ${ }^{2}$ Directorate of Health Services, Shimla, Himachal Pradesh 171 009; \\ ${ }^{3}$ Department of Radiotherapy, All India Institute of Medical Sciences, Rishikesh, Uttarakhand 249 203; \\ ${ }^{4}$ Department of Radiotherapy, Maharishi Markandeshwar University, Ambala, Haryana 133 203, India
}

Received May 28, 2020; Accepted August 24, 2020

DOI: $10.3892 /$ wasj.2020.65

\begin{abstract}
The standard-of-care in locally advanced squamous cell carcinoma of the head and neck (LA SCCHN) remains concurrent chemoradiotherapy. The present study compared the disease response and safety profile of induction chemotherapy followed by concomitant chemoradiotherapy (CRT) vs. CRT alone in patients with LA SCCHN. The present prospective randomized study was conducted between July, 2014 and July, 2015 on 52 patients with SCCHN of the oropharynx, hypopharynx and larynx. Patients were randomly divided into the induction chemotherapy [docetaxel, cisplatin and 5-FU (TPF)] followed by CRT arm (TPF + CRT arm, n=25) or the CRT alone arm (CRT arm, n=27). The disease response, and acute and late toxicities were assessed. At the first follow-up (6 weeks), the overall response rate (ORR) was $82.6 \%$ for the TPF + CRT arm and $72 \%$ for the CRT arm; the difference was not significant. In addition, no statistically significant differences were observed in the nodal response between the treatment arms. Acute toxicities were significantly higher in the TPF + CRT arm, with respect to mucositis and hematological toxicities. No differences were observed in late-onset toxicities observed following 3 months of radiotherapy. Triple drug-based sequential therapy was tolerable in the population in the present study and may thus hold promise for the treatment of SCCHN; however, larger prospective studies are required to confirm these results.
\end{abstract}

Correspondence to: Dr Priyanka Rana, Directorate of Health Services, Shimla, Himachal Pradesh 171 009, India

E-mail: dramitrana1982@gmail.com

Key words: induction chemotherapy followed by chemoradiotherapy, chemoradiotherapy, induction chemotherapy, head and neck cancer, squamous cell carcinoma of the head and neck

\section{Introduction}

Head and neck cancer is the sixth most common type of cancer worldwide (1), with 500,000 new cases diagnosed annually. It constitutes $5 \%$ of all cancer cases worldwide. In India, head and neck cancer accounts for $29.6 \%$ of all cancer cases among males (range, 24.3-34.3\%) and $11.84 \%$ of all cancer cases among females (range, 10.5-15.5\%) as per different hospital-based registries $(2,3)$. Squamous cell carcinoma of the head and neck (SCCHN) constitutes $>90 \%$ of all head and neck cancers (1). In India, SCCHN arising from the oral cavity is the most common type of cancer among males and the third most common type among females $(1,4)$. Approximately, $16 \%$ of all cancer cases were registered as SCCHN at the Regional Cancer Centre, Indira Gandhi Medical College, Shimla, India, from 2001 to 2010, accounting for $10.6 \%$ of all cancer cases among males and $5.4 \%$ of all cancer cases among females (unpublished data). The geographic distribution reveals a very large variation in the incidence of head and neck cancers in different countries, with low incidences reported in Western Europe and high incidences in South Asia, parts of Africa and South America (5).

The incidence of early-stage SCCHN (stage I or II) is 40\%, whereas $60 \%$ of cases are reported with locally advanced (stages III and IVA/B) and metastatic (stage IVC) disease (6). Therapeutic options for early-stage SCCHN include both surgery and radiotherapy as a single treatment modality, with a cure rate of $\sim 80 \%(6,7)$. Radiotherapy alone has long been the standard non-surgical therapy for locally advanced disease $(8,9)$. A previous meta-analysis of individual patient data from $>10,000$ participants in 63 trials [Meta-Analysis of Chemotherapy on Head and Neck Cancer (MACH-NC)] demonstrated that the addition of chemotherapy to radiotherapy in both definitive and adjuvant postoperative settings resulted in a $12 \%$ reduction in the risk of mortality from head and neck cancer, corresponding to an absolute improvement of $4 \%$ in the 5-year survival rates (10).

The use of induction chemotherapy followed by radiotherapy has resulted in organ preservation without compromising overall survival, when compared with radiotherapy alone in the 
treatment of SCCHN (11). Previously, 2 phase III TAX323 (12) and TAX324 (13) trials demonstrated the efficacy of induction chemotherapy [TPF (docetaxel and cisplatin, day 1; fluorouracil by continuous infusion, days 1 to 5)] plus radiotherapy for the treatment of patients with locally advanced (LA) unresectable SCCHN with overall response rates (ORRs) of $68 \%$ and $72 \%$, respectively. The aforementioned studies revealed that a therapeutic gain may be achieved in patients with SCCHN when concomitant chemoradiotherapy is preceded by induction chemotherapy. Hence, the present study compared induction chemotherapy followed by concomitant chemoradiotherapy (CRT) vs. CRT alone in Indian patients with LA SCCHN.

\section{Materials and methods}

Study population. Patients of either sex, aged $\leq 70$ years with a histologically confirmed diagnosis of stage IVA/B SCCHN of the oropharynx, hypopharynx and larynx, who were previously untreated and had a Karnofsky performance status score of $>70$, were included in the present study. The key exclusion criteria were the following: A histology other than SCCHN, hemoglobin ( $\mathrm{Hb})$ levels $\leq 10 \mathrm{gm} \%$, deranged liver and renal function tests and the presence of distant metastasis.

Study design. The present study was a prospective, randomized two-arm study conducted between July, 2014 and July, 2015. The patients $(\mathrm{n}=52)$ were randomly divided by stratification into 2 treatment groups/arms: The induction chemotherapy (docetaxel, cisplatin and 5-FU) followed by CRT arm (TPF + CRT arm; n=25) and the conventional CRT alone arm (CRT arm; n=27). Randomization was carried out by stratification, and the treatment assignment was stratified according to the site of disease (hypopharynx, larynx or oropharynx), $\mathrm{N}$ stage (node -ve or $+\mathrm{ve}$ ) and T stage (T1 and T2 vs. T3 and T4). Patients were randomly divided into the TPF + CRT arm and CRT arm based on the treatment they received. Approximately equal numbers of patients were assigned to each group.

\section{Study treatments}

$T P F+C R T$ arm. Patients randomly divided into the TPF + CRT arm were administered induction chemotherapy with docetaxel $\left(75 \mathrm{mg} / \mathrm{m}^{2}\right)$ on day 1 , and cisplatin $\left(75 \mathrm{mg} / \mathrm{m}^{2}\right)$ and 5 -FU $(750 \mathrm{mg})$ on days 1 and 2 in 3 weekly cycles for a total of 3 cycles. Granulocyte-colony stimulating factor (G-CSF; Filgrastim, $300 \mu \mathrm{g}$ ) was administered prophylactically on day 3 of each cycle. Dexamethasone $(16 \mathrm{mg})$, ranitidine $(50 \mathrm{mg})$, chlorpheniramine maleate $(5 \mathrm{mg}$ ) and ondansetron $(8 \mathrm{mg})$ were administered in each cycle. Dose modifications were allowed as follows: i) The dose of docetaxel was reduced after any episode of febrile neutropenia, grade 4 neutropenia (lasting $>5$ days), grade 4 thrombocytopenia, or $>$ grade 3 asthenia; ii) The dose of cisplatin was reduced to $75 \%$ of the original dose in subsequent cycles if any of the following occurred: > grade 3 sensory neurotoxicity, $\geq$ grade 2 nephrotoxicity, persistent grade 4 neutropenia or neutropenic fever following the dose reduction of docetaxel; iii) The dose of 5-FU was reduced by $25 \%$ in any of the following circumstances: For patients with grade 3 diarrhea lasting for $>7$ days despite the administration of loperamide, mucositis grade 3 lasting for $>5$ days, or grade 4 mucositis.
Following 3 cycles of induction chemotherapy, concurrent CRT with cisplatin $\left(30 \mathrm{mg} / \mathrm{m}^{2}\right)$ on day 1 of each week and conventional radiotherapy daily with $2 \mathrm{~Gy}$ fraction for 5 days a week for a total of $6 \frac{1}{2}$ weeks (total, $66 \mathrm{~Gy} / 6 \frac{1}{2}$ weeks $/ 33 \#$ ) were administered (Fig. 1A).

CRT arm (conventional CRT). Patients assigned to the CRT arm received standard concomitant CRT with cisplatin $\left(30 \mathrm{mg} / \mathrm{m}^{2}\right)$ on day 1 of each week for 7 doses and conventional radiotherapy daily with a 2 Gy fraction for 5 days a week for a total of $6 \frac{1}{2}$ weeks (total, $66 \mathrm{~Gy} / 61 / 2$ weeks/33\#). G-CSF (Filgrastim, $300 \mu \mathrm{g}$ ) was administered only if necessary, after reviewing the investigations, not prophylactically (Fig. 1B).

Study assessments. The first follow-up was performed at 6 weeks following treatment and subsequent follow-ups were performed every 2 months. The primary endpoint was the response rate (RECIST 1.1) at 6 weeks as evaluated by the following criteria: i) Complete response (CR): A complete regression of the lesion (primary, as well as neck nodes); ii) Partial response (PR): A $>50 \%$ regression in the lesion in maximal diameter; iii) Stable disease: If the lesion regressed $<50 \%$ in maximal diameter; and iv) Progressive disease: If the lesion increased by $25 \%$ or the appearance of a new lesion or secondary metastatic disease were noted.

Toxicity profiles were evaluated each week during treatment and at the end of treatment. The toxicity was assessed according to the Radiation Therapy Oncology Group toxicity criteria (14). Treatment toxicities occurring within 90 days of the commencement of radiotherapy were considered acute and those occurring or persisting $>90$ days after the commencement of radiotherapy were considered as late.

Statistical analysis. Quantitative data are presented as the means and standard deviation, and qualitative data by frequency and distribution. The Student's t-test and $\chi^{2}$ test were used for the statistical comparisons of parametric data. Statistical significance was considered as follows: $\mathrm{P}>0.05$ as non-significant, $\mathrm{P}=0.05-0.01$ as significant and $\mathrm{P}<0.01$ as highly significant. SPSS (version 24) was used for statistical analysis.

\section{Results}

Patient disposition and demographics. Of the 52 patients enrolled in the present study, 25 were randomized to the TPF + CRT arm and 27 to the CRT arm (Fig. 2). The median age of the patients was 56.2 years. The patients were followed-up for a median duration of 4 months. The baseline characteristics (Table I) of the study participants did not differ significantly between the 2 study arms. The present study was conducted after due approval from the Institutional Ethics Committee, Indira Gandhi Government Medical College (IGMC), Shimla, Himachal Pradesh, India. All the patients provided written informed consent for participation.

Treatment efficacy. In total, 2 patients in the TPF + CRT and CRT group (in each group) were lost to follow-up. The ORR was $82.6 \%$ [CR, $78.3 \%$ (18/23); PR, 4.3\% (1/23)] for the TPF + CRT arm and $72 \%$ [CR, 64\% (16/25); PR, 8\% (2/25)] for the CRT arm at the first follow-up (Fig. 3). No significant differences 

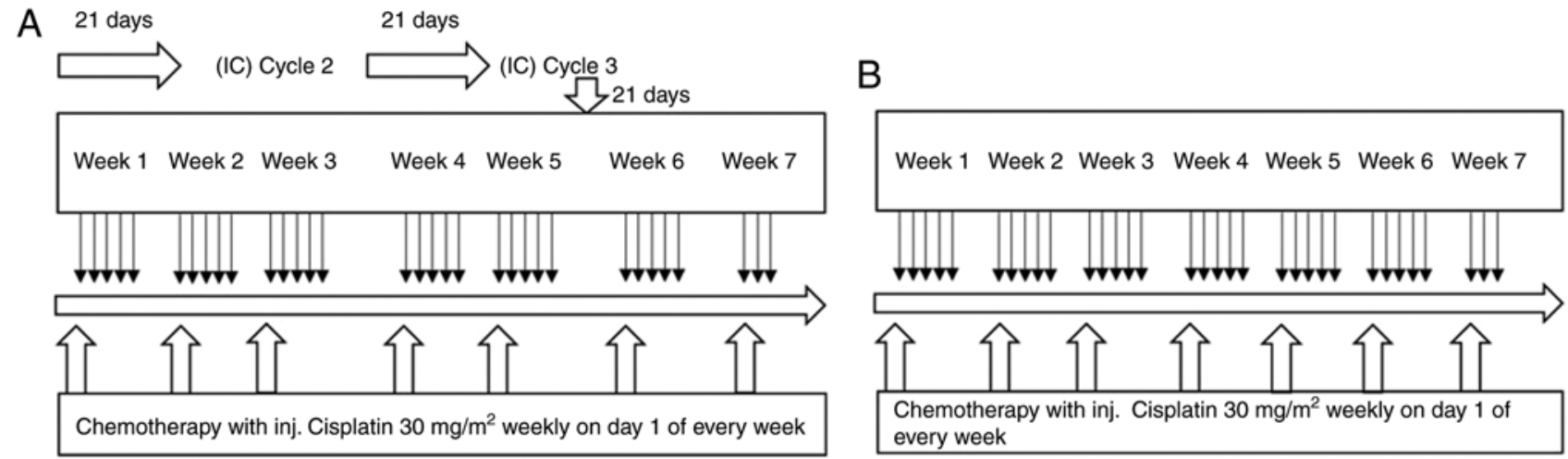

Figure 1. Study design. (A) TPF + CRT arm, (B) CRT arm. In the TPF + CRT arm, following 3 cycles of induction chemotherapy, concurrent chemoradiotherapy with cisplatin $\left(30 \mathrm{mg} / \mathrm{m}^{2}\right)$ on day 1 of each week and conventional radiotherapy daily with 2 Gy fraction for 5 days a week for a total of $61 / 2$ weeks (total, $66 \mathrm{~Gy} / 6 \frac{1}{2}$ weeks/33\#) were administered. In the CRT arm, standard concomitant CRT with cisplatin $\left(30 \mathrm{mg} / \mathrm{m}^{2}\right)$ on day 1 of every week for 7 doses and conventional radiotherapy daily with $2 \mathrm{~Gy}$ fraction for 5 days a week for a total of $61 / 2$ weeks (total, $66 \mathrm{~Gy} / 6 \frac{1}{2}$ weeks/33\#) were administered. TPF + CRT, induction chemotherapy [TPF (docetaxel and cisplatin, day 1; fluorouracil by continuous infusion, days 1 to 5)] plus radiotherapy; CRT, concomitant chemoradiotherapy alone.

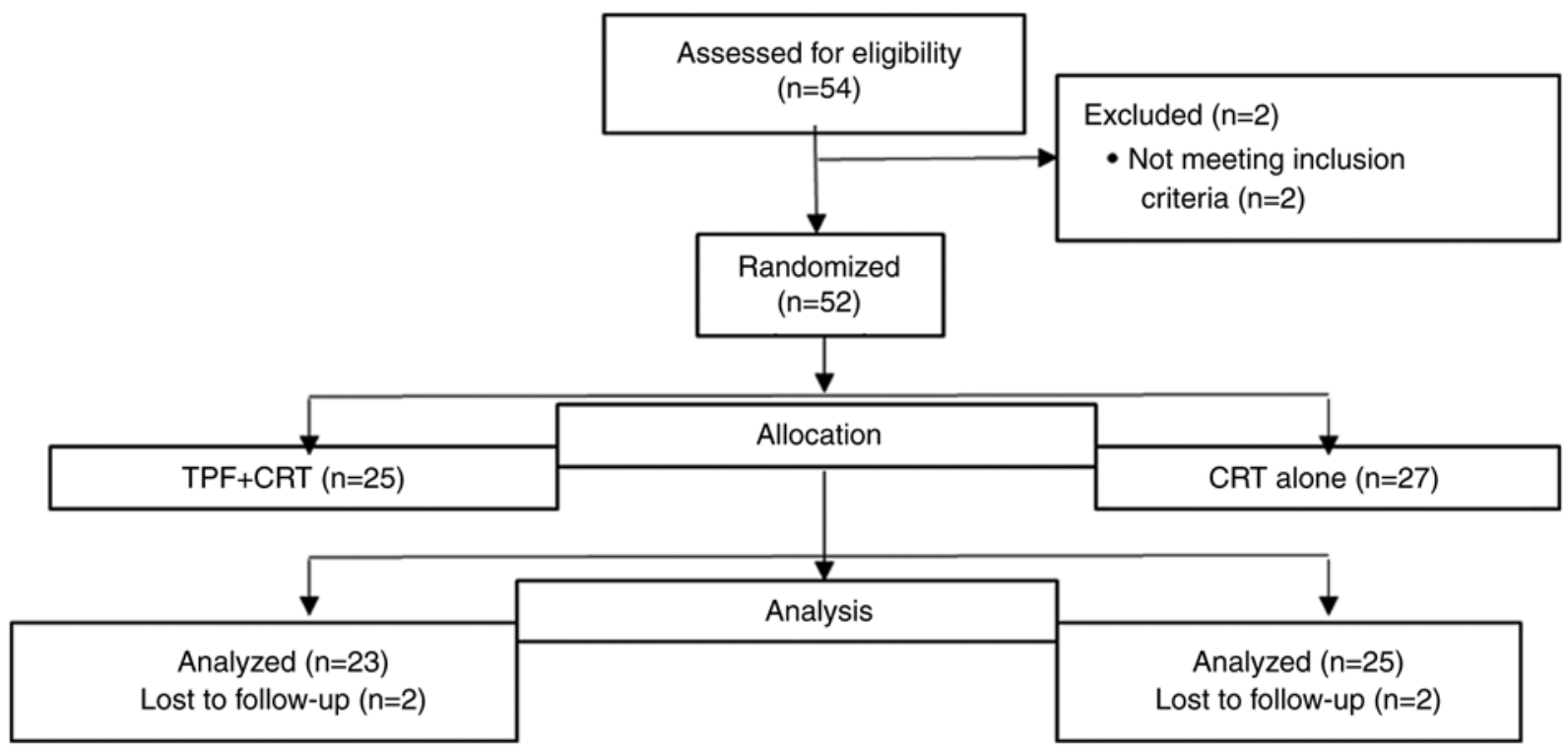

Figure 2. CONSORT flow diagram. Of the 52 patients enrolled in the present study, 25 were randomized to the TPF + CRT arm and 27 were randomized to the CRT arm. TPF + CRT, induction chemotherapy [TPF (docetaxel and cisplatin, day 1; fluorouracil by continuous infusion, days 1 to 5)] plus radiotherapy; CRT, concomitant chemoradiotherapy alone.

$(\mathrm{P}=0.999)$ were observed in disease response at the primary site in both study arms. Disease progression was reported in 1 patient in each arm. The response was not evaluated in 3 and 4 patients in the TPF + CRT and CRT arms, respectively.

For nodal response, the ORR was $82.6 \%$ [CR, 73.9\% (17/23); PR, 8.7\% (2/23)] for the TPF + CRT arm vs. $76 \%$ [CR, $56 \%$ (14/25); PR, 20\% (5/25)] for the CRT arm. No statistically significant differences were observed in nodal response between the treatment arms at the first follow-up.

At the median follow-up of 3.5 months, 17 (73.9\%) patients in the TPF + CRT arm and $15(60 \%)$ patients in the CRT arm achieved CR ( $\mathrm{P}=0.307)$. Similarly, complete nodal response at the median follow-up of 3.5 months was achieved in 16 (69.6\%) and $13(52 \%)$ of the patients in the TPF + CRT and CRT arms, respectively $(\mathrm{P}=0.367)$.

No statistically significant differences were found in $\mathrm{CR}$ in both the treatment arms when analyzed for different subgroups: Sex, smoking status, alcohol status, smoking and alcoholic status, diet, cancer site and cancer stage. A trend for a better response with TPF + CRT was observed across cancer sites (oropharynx, larynx and hypopharynx) and cancer stages (IVA and IVB), although the difference was not statistically significant (Table II).

Safety. During treatment, majority of the patients suffered from grade 3 skin toxicity, which was higher with TPF + CRT as compared to CRT (65.2 vs. $48 \%$; $\mathrm{P}=0.262$ ). Grade 3 mucositis was significantly $(\mathrm{P}=0.016)$ higher in the patients in the TPF + CRT arm (52.2\%) compared to those in the CRT arm (16\%). Grade 2 laryngeal toxicities were observed in the majority of patients, including hoarseness or whispered speech, throat pain and cough. Grade 2 and 3 pharyngeal toxicities combined were higher in the CRT (grade 2,64\%; grade $3,24 \%$ ) vs. the TPF + CRT arm (grade 2, 60.7\%; grade 3, 
Table I. Baseline characteristics of the study participants.

\begin{tabular}{|c|c|c|}
\hline Parameter & $\mathrm{TPF}+\mathrm{CRT}(\mathrm{n}=25)$ & CRT $(n=27)$ \\
\hline Age, mean, years & 54.9 & 60.7 \\
\hline Median & 56 & 60 \\
\hline Range & $37-70$ & $45-70$ \\
\hline 31-40 years, n (\%) & $1(4)$ & 0 \\
\hline $41-50$ years, $\mathrm{n}(\%)$ & $6(24)$ & $6(22.2)$ \\
\hline $51-60$ years, $\mathrm{n}(\%)$ & $7(28)$ & $8(29.6)$ \\
\hline $61-70$ years, $\mathrm{n}(\%)$ & $11(44)$ & $13(48.2)$ \\
\hline \multicolumn{3}{|l|}{ Sex, n $(\%)$} \\
\hline Male & $23(92)$ & $26(96.3)$ \\
\hline Female & $2(8)$ & $1(3.7)$ \\
\hline Smoker, n (\%) & $22(88)$ & $26(96.2)$ \\
\hline \multicolumn{3}{|l|}{ Alcohol consumption n (\%) } \\
\hline Chronic alcohol consumption & $8(32)$ & $13(48.1)$ \\
\hline Occasional alcohol consumption & $9(36)$ & $10(37.1)$ \\
\hline No alcohol consumption & $8(32)$ & $4(14.8)$ \\
\hline Karnofsky performance status, mean (range) & $87.6(80-90)$ & $85.9(80-90)$ \\
\hline Hemoglobin levels (g/dl), mean & 12.9 & 12.7 \\
\hline \multicolumn{3}{|l|}{ Cancer sites, $\mathrm{n}(\%)$} \\
\hline Oropharynx & $15(60)$ & $14(51.9)$ \\
\hline Larynx & $7(28)$ & $7(25.9)$ \\
\hline Hypopharynx & $3(12)$ & $6(22.2)$ \\
\hline \multicolumn{3}{|l|}{ Cancer subsites, $\mathrm{n}(\%)$} \\
\hline Vallecula & $6(24)$ & $6(22.2)$ \\
\hline Base of tongue & $7(28)$ & $4(14.8)$ \\
\hline Supraglottis & $5(20)$ & $5(18.5)$ \\
\hline Tonsil & $2(8)$ & $4(14.8)$ \\
\hline Pyriform sinus & $2(8)$ & $4(14.8)$ \\
\hline Glottis & $2(8)$ & $2(7.4)$ \\
\hline Lateral pharyngeal wall & $1(4)$ & $1(3.7)$ \\
\hline Posterior pharyngeal wall & 0 & $1(3.7)$ \\
\hline \multicolumn{3}{|l|}{ T stage, n (\%) } \\
\hline $\mathrm{T} 1$ & $2(8)$ & $2(7.4)$ \\
\hline $\mathrm{T} 2$ & $10(40)$ & $8(29.6)$ \\
\hline T3 & $6(24)$ & $8(29.6)$ \\
\hline $\mathrm{T} 4$ & $7(28)$ & $9(33.3)$ \\
\hline \multicolumn{3}{|l|}{ N stage, n (\%) } \\
\hline N0 & $2(8 \%)$ & $3(11.1 \%)$ \\
\hline $\mathrm{N} 1$ & $3(25 \%)$ & $4(14.81 \%)$ \\
\hline $\mathrm{N} 2$ & $17(68 \%)$ & $18(66.7 \%)$ \\
\hline N3 & $3(12 \%)$ & $2(7.4 \%)$ \\
\hline \multicolumn{3}{|l|}{ Cancer stage, n (\%) } \\
\hline IVA & $20(80)$ & $22(81.5)$ \\
\hline IVB & $5(20)$ & $5(18.5)$ \\
\hline
\end{tabular}

P-value $>0.05$ for all parameters between the 2 groups. TPF + CRT, induction chemotherapy [TPF (docetaxel and cisplatin, day 1 ; fluorouracil by continuous infusion, days 1 to 5)] plus radiotherapy; CRT, concomitant chemoradiotherapy alone.

$17.4 \%$ ), although no significant differences were observed. Significantly higher grade 3 hematological toxicities were observed in the TPF + CRT vs. the CRT arm (60.9 vs. $24 \%$,
$\mathrm{P}=0.012)$. Gastrointestinal toxicity was slightly higher in the TPF + CRT (28\%) vs. the CRT (25.9\%) arm. Febrile neutropenia was observed in $1(4 \%)$ patient receiving TPF + CRT. 


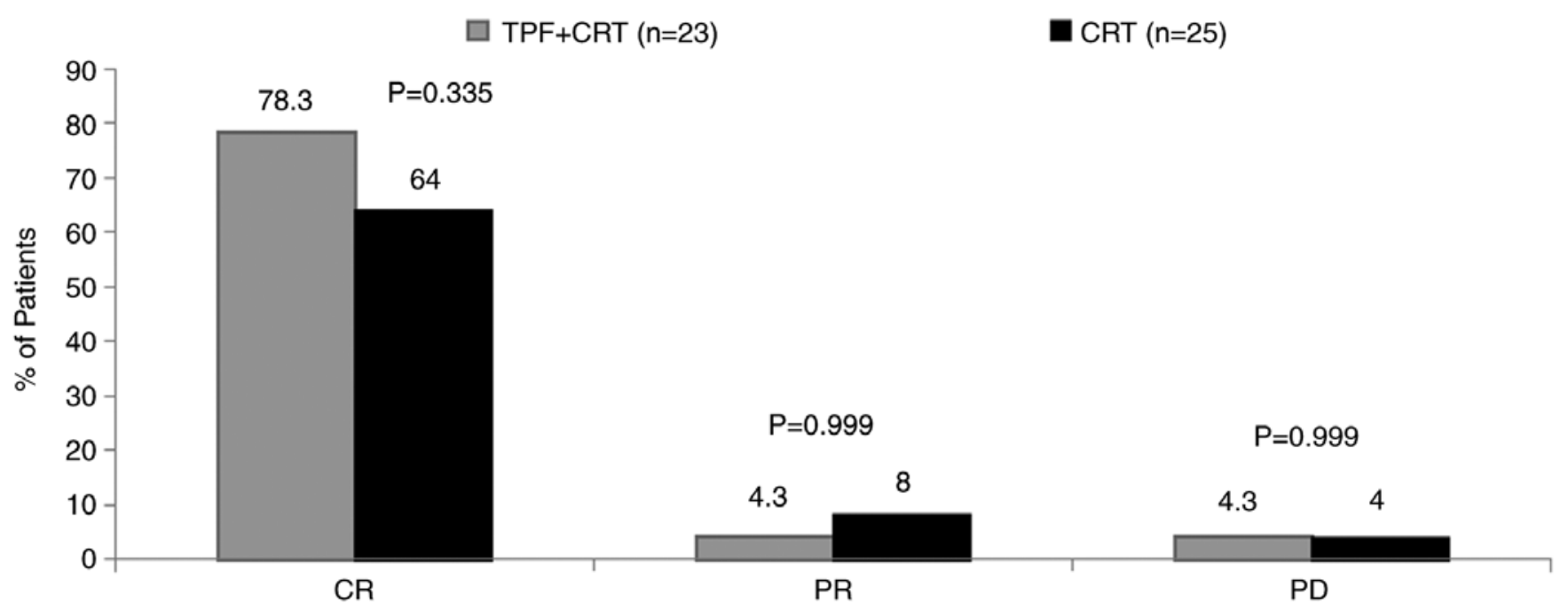

Figure 3. Efficacy evaluation for TPF + CRT vs. CRT arms at first follow-up. $\chi^{2}$ tests were used for statistical comparisons of the parametric data. Statistical significance was as follows: $\mathrm{P}>0.05$ as non-significant, $\mathrm{P}=0.05-0.01$ as significant and $\mathrm{P}<0.01$ as highly significant. $\mathrm{CR}$, complete response; $\mathrm{PD}$, progressive disease; PR, partial response; TPF + CRT, induction chemotherapy [TPF (docetaxel and cisplatin, day 1; fluorouracil by continuous infusion, days 1 to 5)] plus radiotherapy; CRT, concomitant chemoradiotherapy alone.

Treatment interruptions (TPF + CRT, 40\%; CRT, 37\%) were observed as follows: TPF + CRT arm: Due to grade 3 and 4 skin toxicity, grade 3 and 4 mucositis and hematological toxicity; CRT arm: Due to the withdrawal of consent and 1 patient leaving the treatment in between.

Toxicity at first follow-up. In the TPF + CRT arm, $65.2 \%$ of the patients had grade 1 salivary gland toxicity compared with $36 \%$ patients in the CRT arm. Skin reactions occurring during the treatment were healed at first follow-up. Mucositis was not healed in 1 patient $(4.34 \%)$ in the TPF + CRT arm and 2 patients (8\%) in the CRT arm. The majority of the patients had grade 1 salivary gland toxicity (Table III).

Late skin toxicity, depigmentation. Depigmentation was present in $16(69.6 \%)$ of the patients in the TPF + CRT arm compared with $14(56 \%)$ of patients in the CRT arm. Subcutaneous fibrosis was present in $13 \%$ of the patients in the TPF + CRT arm compared with $12 \%$ in the CRT arm (Table III).

\section{Discussion}

Concomitant chemoradiation has demonstrated an $8 \%$ absolute survival advantage in the meta-analysis conducted by Pignon et al, and has now become the standard of care in LA SCCHN cancers (10). The combination of induction chemotherapy with concomitant CRT for the treatment of SCCHN has been examined in several clinical trials. Browman et al reported a pooled analysis of 18 randomized controlled trials (RCTs) in 3,192 patients, in which concomitant chemotherapy-radiation therapy was compared to radiation therapy alone. Overall, the chemotherapy-radiation therapy arm was superior for the reduction in mortality compared with radiation therapy alone $(\mathrm{P}<0.0001)$. The results further demonstrated that platinum-based concomitant CRT is superior to conventional radiotherapy alone in improving survival in locally advanced SCCHN (15).
The current randomized prospective study compared the combination of induction chemotherapy with the TPF regimen followed by concurrent CRT vs. CRT alone in 52 patients with advanced SCCHN at the Regional Cancer Centre in India. The study demonstrated a higher local control at primary and nodal sites with TPF + CRT as compared with CRT alone, though the difference was not statistically significant. The ORR was reported in $82.6 \%$ patients with TPF + CRT vs. $72 \%$ with CRT.

The TAX323 (12) trial in patients with advanced SCCHN (stages III and IV) with an unresectable disease demonstrated an ORR of 68\% with TPF + CRT regimen. The TAX 324 (13) trial reported loco-regional control in $72 \%$ patients with both resectable and unresectable advanced SCCHN (stage III or IV) who were treated with 3-cycles of induction chemotherapy with TPF regimen (docetaxel, cisplatin and 5-FU) followed by concomitant radiotherapy. The phase III EORTC trial of TPF followed by radiotherapy in unresectable LA SCCHN $(n=177)$ patients showed a response rate of $67.8 \%$ (16). The GSTTC trial that compared the induction TPF followed by concomitant treatment $(\mathrm{n}=206)$ vs. concomitant treatment alone (208) in patients with LA SCCHN revealed an ORR of $76 \%$ with induction regimen; the CRs were significantly higher in the induction chemotherapy arm (42.5\% vs. $28 \%, \mathrm{P}=0.0028)(17)$. The efficacy results of these studies are comparable to those reported in the present study.

The efficacy of TPF + CRT ( $=50)$ vs. CRT alone $(n=51)$ in patients with LA SCCHN was evaluated by Paccagnella et al in a European population (18). CR was reported in 50\% patients in the TPF + CRT arm vs. $21.2 \%$ in the CRT arm, compared to 78.3 and $64 \%$, respectively as observed in the present study. On subset analysis, a trend for a better response was observed with TPF + CRT vs. CRT across the larynx, oropharynx and hypopharynx in the present study, although the results were not statistically significant.

In the present study, confluent fibrinous mucositis with pain (grade 3 acute mucositis) and grade 4 mucositis was observed 
Table II. Subgroup evaluation for both groups.

\begin{tabular}{|c|c|c|c|c|c|}
\hline \multirow[b]{2}{*}{ Parameter } & \multicolumn{2}{|c|}{$\mathrm{TPF}+\mathrm{CRT}(\mathrm{n}=23)$} & \multicolumn{2}{|c|}{ CRT $(n=25)$} & \multirow[b]{2}{*}{ P-value } \\
\hline & $\mathrm{CR}(\%)$ & No CR $(\%)$ & $\mathrm{CR}(\%)$ & No CR $(\%)$ & \\
\hline \multicolumn{6}{|l|}{ Sex } \\
\hline Male & $18(85.7)$ & $3(14.3)$ & $16(66.7)$ & $8(33.3)$ & 0.177 \\
\hline Female & $1(50)$ & $1(50)$ & 0 & $1(100)$ & 0.999 \\
\hline Smoker & $15(75)$ & $5(25)$ & $16(64)$ & $9(36)$ & 0.059 \\
\hline Alcohol consumption, n (\%) & $13(81.25)$ & $3(18.8)$ & $15(68.1)$ & $7(31.8)$ & 0.469 \\
\hline No alcohol consumption, $\mathrm{n}(\%)$ & $5(71.4)$ & $2(28.6)$ & $2(66.7)$ & $1(33.3)$ & 0.999 \\
\hline Smoking and alcohol consumption, n (\%) & $12(80)$ & $3(20)$ & $15(68.2)$ & $7(31.8)$ & 0.481 \\
\hline Non-vegetarian, n (\%) & $18(90)$ & $2(10)$ & $14(63.6)$ & $8(36.4)$ & 0.071 \\
\hline Vegetarian, n (\%) & $2(66.7)$ & $1(33.3)$ & $2(66.7)$ & $1(33.3)$ & 0.999 \\
\hline \multicolumn{6}{|l|}{ Site } \\
\hline Oropharynx & $11(78.6)$ & $3(21.4)$ & $8(61.5)$ & $5(38.5)$ & 0.420 \\
\hline Larynx & $4(80)$ & $1(20)$ & $3(50)$ & $3(50)$ & 0.546 \\
\hline Hypopharynx & $2(66.7)$ & $1(33.3)$ & $3(50)$ & $3(33.3)$ & 0.999 \\
\hline \multicolumn{6}{|l|}{ Cancer stage } \\
\hline IVA & $13(72.2)$ & $5(27.8)$ & $13(65)$ & $7(35)$ & 0.633 \\
\hline IVB & $4(80)$ & $1(20)$ & $3(60)$ & $2(40)$ & 0.999 \\
\hline
\end{tabular}

TPF + CRT, induction chemotherapy [TPF (docetaxel and cisplatin, day 1; fluorouracil by continuous infusion, days 1 to 5)] plus radiotherapy; CRT, concomitant chemoradiotherapy alone; CR, complete response.

Table III. Toxicity profile at first follow-up.

\begin{tabular}{lcc}
\hline Parameter & TPF + CRT $(\mathrm{n}=23)(\%)$ & CRT $(\mathrm{n}=25)(\%)$ \\
\hline Skin toxicities & $20(87)$ & $20(80)$ \\
No toxicity & & $18(78.3)$ \\
Mucositis & $19(82.6)$ & $2(8)$ \\
No toxicity & $1(4.34)$ & 0.612 \\
Grade 1 & & $11(44)$ \\
Salivary Gland Toxicity & $4(17.4)$ & $9(36)$ \\
No toxicity & $15(65.2)$ & 0.458 \\
Grade 1 & $1(4.3)$ & $14(56)$ \\
Grade 2 & $16(69.6)$ & $3(12)$ \\
Late Toxicity: Depigmentation & $3(13.0)$ & 0.999 \\
Late Toxicity: Subcutaneous Fibrosis & & 0.054 \\
Late Toxicity: Salivary gland & $3(13.0)$ & 0.999 \\
No toxicity & $19(82.6)$ & 0.376 \\
Grade 1 & $1(4.34)$ & $21(84)$ \\
Grade 2 & & $1(4)$ \\
\hline
\end{tabular}

TPF + CRT, induction chemotherapy [TPF (docetaxel and cisplatin, day 1; fluorouracil by continuous infusion, days 1 to 5)] plus radiotherapy; $\mathrm{CRT}$, concomitant chemoradiotherapy alone.

more often in the TPF + CRT arm (52\%) as compared with the CRT arm $(18.5 \%)$ during radiation treatment $(\mathrm{P}=0.016)$. Febrile neutropenia was reported in 1 patient with TPF + CRT. In the DeCIDE trial, the most common grade 3/4 toxicities during induction chemotherapy (TPF + CRT arm) were febrile neutropenia (11\%) and mucositis (9\%) (19). In the present study, a higher trend for skin, laryngeal and GI toxicities was observed with TPF + CRT compared with CRT. Furthermore, a higher 
trend for acute toxicities was observed with the TPF + CRT regimen with significant differences observed for mucositis and hematological toxicities. Grade 3/4 hematological toxicity was markedly higher with TPF + CRT. The acute toxicity results in the present study were comparable to those observed in the DeCIDE, PRADIGM and GSTCC trials $(17,19,20)$. None of the patients in the present study exhibited any skin reactions at the first follow-up and all previous skin reactions were healed completely. However, 1 patient in the TPF + CRT arm and 2 patients in the CRT arm had mucositis and were still healing even after 6 weeks of completion of radiation therapy at the first follow-up; however, at the second follow-up up, all acute toxicities in patients of both the arms were completely healed. A similar trend for higher late toxicities in the form of subcutaneous fibrosis was observed in the TPF + CRT arm as compared with the CRT arm, although the difference in late toxicities was not statistically significant.

Some limitations of the present study should be mentioned. These include the small sample size and the unavailability of the progression-free survival (PFS) and overall survival (OS) data.

In conclusion, local and nodal response at first follow-up was higher with TPF + CRT regimen compared with CRT, although the difference was not statistically significant. The toxicities were higher in the TPF + CRT arm with significant differences for mucositis and hematological toxicities. However, at the first follow-up, mucositis was present in only 1 patient in the TPF + CRT arm vs. 2 patients in the CRT arm. Although in the present study, no statistically significant differences were observed in late toxicities, a longer follow-up time is required to draw any meaningful conclusion. The present study demonstrates the feasibility of sequential therapy in the management of locally advanced head and neck cancer in the Indian population. Triple drug-based sequential therapy was tolerable in the Indian population context. Further large scale studies with longer follow-up times are warranted to confirm these results in Indian patients and in other populations.

\section{Acknowledgements}

Not applicable.

\section{Funding}

No funding was received.

\section{Availability of data and materials}

The datasets used and/or analyzed during the current study are available from the corresponding author on reasonable request.

\section{Authors' contributions}

AR and PR performed the research, were involved in the acquisition of data, critically revised the manuscript for important intellectual content, and approved the final manuscript. AR, PR, ManojG, RS and ManishG designed the study, were involved in the data interpretation, critically revised the manuscript for important intellectual content, and approved the final manuscript. All authors made substantial contributions to the present study, read and approved the final manuscript and agree to be accountable for all aspects of the work.

\section{Ethics approval and consent to participate}

The present study was conducted after due approval from the Institutional Ethics Committee, Indira Gandhi Government Medical College (IGMC), Shimla, Himachal Pradesh, India. All the patients provided written informed consent for participation.

\section{Patient consent for publication}

Not applicable.

\section{Competing interests}

The authors declare that they have no competing interests.

\section{References}

1. Vigneswaran $\mathrm{N}$ and Williams MD: Epidemiologic trends in head and neck cancer and aids in diagnosis. Oral Maxillofac Surg Clin North Am 26: 123-141, 2014.

2. Indian Council of Medical Research. National Cancer Registry Programme: Ten year consolidated report of the hospital based cancer registries 1984-1993. New Delhi. https://ncdirindia.org/ NCRP/Old_Reports/HBCR_TEN/inside_pages.pdf. Accessed July 10,2014 .

3. Dhull AK, Atri R, Dhankhar R, Chauhan AK and Kaushal V: Major risk factors in head and neck cancer: A retrospective analysis of 12-year experiences. World J Oncol 9: 80-84, 2018.

4. Warnakulasuriya S: Global epidemiology of oral and oropharyngeal cancer. Oral Oncol 45: 309-316, 2009.

5. Gupta N, Gupta R, Acharya AK, Patthi B, Goud V, Reddy S, Garg A and Singla A: Changing trends in oral cancer-a global scenario. Nepal J Epidemiol 6: 613-619, 2016.

6. Szturz P and Vermorken JB: Treatment of elderly patients with squamous cell carcinoma of the head and neck. Front Oncol 6: 199,2016

7. Marur S and Forastiere AA: Head and neck squamous cell carcinoma: Update on epidemiology, diagnosis, and treatment. Mayo Clin Proc 91: 386-396, 2016.

8. Forastiere A, Koch W, Trotti A and Sidransky D: Head and neck cancer. N Engl J Med 345: 1890-1900, 2001.

9. Posner MR, Haddad RI, Wirth L, Norris CM, Goguen LA, Mahadevan A, Sullivan C and Tishler RB: Induction chemotherapy in locally advanced squamous cell cancer of the head and neck: Evolution of the sequential treatment approach. Semin Oncol 31: 778-785, 2004.

10. Pignon JP, Bourhis J, Domenge $C$ and Designe L: Chemotherapy added to locoregional treatment for head and neck squamous-cell carcinoma: Three meta-analyses of updated individual data. MACH-NC Collaborative Group. meta-analysis of chemotherapy on head and neck cancer. Lancet 355: 949-955, 2000.

11. Dietz A, Wiegand S, Kuhnt T and Wichmann G: Laryngeal preservation approaches: Considerations for new selection criteria based on the DeLOS-II trial. Front Oncol 9: 625, 2019.

12. Vermorken JB, Remenar E, van Herpen C, Gorlia T, Mesia R, Degardin M, Stewart JS, Jelic S, Betka J, Preiss JH, et al: Cisplatin, fluorouracil, and docetaxel in unresectable head and neck cancer. N Engl J Med 357: 1695-1704, 2007.

13. Posner MR, Hershock DM, Blajman CR, Mickiewicz E, Winquist E, Gorbounova V, Tjulandin S, Shin DM, Cullen K, Ervin TJ, et al: Cisplatin and fluorouracil alone or with docetaxel in head and neck cancer. N Engl J Med 357: 1705-1715, 2007.

14. Cox JD, Stetz J and Pajak TF: Toxicity criteria of the Radiation therapy oncology group (RTOG) and the European organization for research and treatment of cancer (EORTC). Int J Radiat Oncol Biol Phys 31: 1341-1346, 1995. 
15. Browman GP, Hodson DI, Mackenzie RJ, Bestic N and Zuraw L; Cancer Care Ontario Practice Guideline Initiative Head and Neck Cancer Disease Site Group: Choosing a concomitant chemotherapy and radiotherapy regimen for squamous cell head and neck cancer: A systematic review of the published literature with subgroup analysis. Head Neck 23: 579-589, 2001.

16. Vermorken JB, Remenar E, ven Herpen C, Lluch JG, Stewart S, Gorlia T, Degardin TG, Schollen K and Bernier J: Standard cisplatin/infusional 5-fluorouracil (PF) vs. docetaxel (T) plus PF (TPF) as neoadjuvant chemotherapy for nonresectable locally advanced squamous cell carcinoma of the head andneck (LA-SCCHN): A phase III trial of the EORTC head and neck cancer group (EORTC \#24971). J Clin Oncol 22 (14 Suppl): S5508, 2004.

17. Ghi MG, Paccagnella A, Ferrari D, Foa P, Alterio D, Codeca C, Nole F, Verri E, Orecchia R, Morelli F, et al: Induction TPF followed by concomitant treatment versus concomitant treatment alone in locally advanced head and neck cancer. A phase II-III trial. Ann Oncol 28: 2206-2212, 2017.
18. Paccagnella A, Ghi MG, Loreggian L, Buffoli A, Koussis H, Mione CA, Bonetti A, Campostrini F, Gardani G, Ardizzoia A, et al: Concomitant chemoradiotherapy versus induction docetaxel, cisplatin and 5 fluorouracil (TPF) followed by concomitant chemoradiotherapy in locally advanced head and neck cancer: A phase II randomized study. Ann Oncol 21: 1515-1522, 2010.

19. Cohen EE, Karrison TG, Kocherginsky M, Mueller J, Egan R, Huang CH, Brockstein BE, Agulnik MB, Mittal BB, Yunus F, et al: Phase III randomized trial of induction chemotherapy in patients with N2 or N3 locally advanced head and neck cancer. J Clin Oncol 32: 2735-2743, 2014.

20. Haddad R, O'Neill A, Rabinowits G, Tishler R, Khuri F, Adkins D, Clark J, Sarlis N, Lorch J, Beitler JJ, et al: Induction chemotherapy followed by concurrent chemoradiotherapy (sequential chemoradiotherapy) versus concurrent chemoradiotherapy alone in locally advanced head and neck cancer (PARADIGM): A randomised phase 3 trial. Lancet Oncol 14: 257-264, 2013.

(i) $\Theta$ This work is licensed under a Creative Commons Attribution-NonCommercial-NoDerivatives 4.0 International (CC BY-NC-ND 4.0) License. 